\title{
Using Self-handicaps to Protect Self-esteem: Young Peoples' Responses to Evaluative Threat in Sport
}

\author{
P.D. Cooley ${ }^{*}$, S.J. Pedersen \\ Human Movement, Faculty of Education, University of Tasmania, Launceston, Tasmania, 7248 Australia \\ *Corresponding Author: dean.cooley@utas.edu.au
}

Copyright $@ 2014$ Horizon Research Publishing All rights reserved.

\begin{abstract}
The purpose of this investigation was to examine the relationship between self-esteem, certainty of self-esteem and trait self-handicapping and the use of self-avowals of performance disruption. A random sample of young people for whom sport was highly self-relevant, were randomly allocated to a condition that exposed them to unexplained failure on a sport test or a non-evaluative condition. Results showed that low self-esteem individuals who experienced unexpected failure significantly reported more performance disruption as a self-handicap. Trait self-handicapping, certainty of self-esteem and gender were not predictors of self-handicapping. Recommendations for teachers and coaches are discussed.
\end{abstract}

Keywords Self-Handicapping, Self-Esteem, Sport

\section{Introduction}

Protecting self-esteem is a fundamental human characteristic. One protective ploy is the use of self-handicapping strategies, which are a priori barriers designed to obfuscate evaluation of ability [1]. Self-handicaps encompass behaviors such as effort withdrawal and reports of impediments such as illness as a reason for failure on a task [2]. Thus, if failure occurs on a task it can be attributed to the self-handicap, and not a lack of ability [3]. Yet, if success occurs the individual can claim additional credit for succeeding at the task despite the presence of the self-handicap. Self-handicapping presumably occurs because there are threats to self-esteem on important self-relevant dimensions [3], and individual differences affect the tendency to self-handicap. Yet this evidence is restricted to self-esteem that is relevant to academic based self-concepts of adults and college populations [4]. This study explored differences in self-handicapping as a function of self-esteem on a self-relevant evaluative assessment.

Individual differences in self-esteem are associated with differentials in self-handicapping tendencies [5-12]. Self-esteem is the attitude towards the self, whereas self-esteem certainty refers to the short-term fluctuations that people experience in their baseline attitudes towards self-esteem [13] Theoretically, the variable of certainty of self-esteem should be a more robust indicator of the tendency to self-handicap than level of self-esteem [12], yet the relationship is obscure with differences evident for both level and certainty of self-esteem. Regardless of level of self-esteem, it seems that low certainty self-esteemed individuals when faced with evaluated threat engage in more behavioral self-handicapping (i.e., withdraw effort) than those more certain of their esteem [5]. Yet, when self-esteem is conceptualized as high and low other differences emerge [8].

The consensus is that high self-esteemed individuals self-handicap more than low self-esteemed individuals [8-9] Yet, Tice [8] using a sham test which involved completing a physical skill task showed that in performance uncertainty situations where evaluative low scores are meaningful, low self-esteemed individuals, self-handicapped. Alternatively, in situations when a high score was meaningful, high self-esteemed individuals self-handicapped. These results suggest that low-esteemed individuals self-handicap for esteem protection, rather than to enhance success. Yet the applicability of these results to sport and self-handicapping is limited because the nature of the task used by Tice [8-9] was ambiguous [8-9]. That is, the researchers instructed participants that the outcome of the physical task was related to an assessment of non-verbal intelligence.

Despite a body of literature [5-12] devoted to describing how individual differences in self-esteem affect the tendency to self-handicap there is scant evidence that describes the relationship between self-esteem and self-handicapping. Prapavessis and Grove [11] explored the relationship between self-esteem and the trait of self-handicapping and the use of self-reports of impediments as a means of self-handicapping. Specifically, on a physical task where the outcome had importance in terms of diagnosticity of ability, low self-esteemed participants who were classified as high trait excuse-makers, self-handicapped significantly more than other participants. Despite this finding, the evidence is somewhat compromised because the research design did not incorporate random assignment and there was no 
experimental manipulation of performance uncertainty.

Studies that have manipulated performance certainty [i.e., 8, 14-17] to assess self-handicapping behavior in the presence of evaluative threat have followed a design that can be abbreviated as $\mathrm{A} * \mathrm{~B}$ [14]. The $\mathrm{A}$ represents an evaluative test of an important ability and $\mathrm{B}$ is a parallel test. Measurement of the dependent variable occurs between A and B and after the delivery of performance scores. Typically, participants do not complete test B because the dependent variable has been already been collected. Researchers use a variety of deceptive strategies to manipulate participants' performance certainty. These include but are not limited to modifying the test so that participants are unable to discern their true performances [14]. To create performance uncertainty, researchers then give participants non-contingent bogus performance scores associated with the first test attempt [i.e., 15-17]. The bogus performance scores are theorized to manipulate participants' performance certainties. For example, on a test which appears to be easy, participants are told that they have failed on the first test.

Participants exposed to the manipulation (sham test + bogus performance scores) after completing test A should have a high degree of uncertainty about performing successfully in test B given the non-contingency of the performance scores. Consequently, these participants would be most likely to use self-handicaps to externalize the reasons for a possible flawed performance in test B. On the other hand, participants not exposed to the manipulation (control) would have no reason to doubt their capacity to perform successfully as the evaluative threat would be minimized because they receive no performance data. Therefore, they would have no reason to use a self-handicap to protect against the implications of possible flawed performance on test B. Thus, if there is no manipulation of performance certainty, it is unclear as to the relationship between self-handicapping and any individual difference variable such as self-esteem.

Finally, within the framework of individual differences, the use of self-handicapping can also be differentiated on the basis of gender. Research has repeatedly found a gender difference with males self-handicapping to a greater degree than females. Yet the reason for this difference remains elusive [18]. Moreover, in some regards, these differences disappear when the achievement domain is sport and for different forms of self-handicapping (i.e., behavioral versus self-avowals) $[1,4,11]$.

In sum, the evidence for an anticipated relationship between self-esteem and self-handicapping is unclear for self-relevant tasks associated with sport. To the knowledge of the authors, no research describes if individual differences in level or certainty of self-esteem affect the tendency to self-handicap when young people are faced with evaluative threat associated with? sport. Consequently, this investigation explored if individual differences in self-esteem and certainty of self-esteem were predictive of self-handicapping under evaluative threat conditions. Further, an aim of this investigation was to explore if the trait of self-handicapping, which is the tendency to use self-handicaps when performance uncertainty exists, added to the predictability of self-handicapping. Self-handicapping was operationalized as self-avowals of impediments causing performance disruption before a test of ability. The research aim was achieved by exploring the relationship between the three independent variables (self-esteem, certainty of self-esteem, and trait self-handicapping) and the dependent variable of self-reported performance disruption. Moreover, the relationship was explored within the context of a non-contingent failure group and a non-evaluative group.

Based on previous research [5-12] it was hypothesized that level of self-esteem, certainty of self-esteem, and trait self-handicapping would be significant predictors of self-handicapping in an esteem threatening evaluative situation. All procedures in the study were approved by the ethical committee at Victoria University of Technology, Australia.

\section{Materials and Methods}

\subsection{Participants}

A total of 321 athletes from 15 different sports replied to an email invitation to participate in the study. All participants indicated on a 7-point scale ( $1=$ not important, $7=$ very important $)$ that success at sport was important to them $(M=5.9, S D \pm 0.65)$. Previous research indicated that individuals tend not to self-handicap when there is a lack of relevance associated with the outcome [11]. One hundred and sixty young athletes (males $n=80$, females $n=80$ ) were randomly selected via a computer random selection process so that equal numbers in each age group and gender $(n=20)$. Athletes played a variety of sports, which included Australian football, basketball, cricket, soccer, softball, and netball. All participants were English speaking.

\subsection{Materials}

\subsubsection{The experimental task}

In this study, the task was a physical test of overarm throwing ability based on Ulrich's Fundamental Motor Skill Assessment [19]. The test was portrayed to participants as a general sport talent identification sport test that was predictive of athletic ability and hence future success in sport. Sham tests need to be modified to an extent where participants do not suspect the sham [15]. The sham test needed to have a degree of novelty so that participants would not become suspicious of their deceptive performance scores. For example, students would be familiar with the overarm throwing test and their previous performance on the test as it is used widely in Tasmanian high schools during physical education. To improve the odds of the bogus test scores manipulating participants' certainty of performance modifications to the test included: represented the test as talent identification instrument that 
had high reliability and validity in predicting athletic skill and future success and the addition of a scoring system, and a screen (1 meter high) to obscure the participants' view of their efforts to minimize feedback and enhance the effect of the bogus performance score manipulation. Participants were instructed to throw a ball overarm at a horizontal target located five meters away, with the aim to score as many points as possible from five attempts. Scoring was based on the ball hitting one of five concentric rings that made up the target.

\subsubsection{Performance certainty.}

To obtain a clearer picture of the manipulation's effectiveness in changing participants' perceptions of certainty, pre-test and post-test measures of certainty were used. Participants in this study responded to the stem, "With regard to the upcoming test, how certain are you that you will have success in the test?" Certainty was measured on a continuous scale with anchors at 1 (no certainty) and 5 (high certainty). Participants completed the measure before test $\mathrm{A}$ and after the feedback contingency manipulation. For participants in the non-evaluative condition, the word 'trial' was substituted for the word 'test' to reduce evaluative threat.

\subsubsection{Performance disruption.}

Self-handicapping was operationalized as performance disruption. Before the second test, participants reported any conditions (listed on a checklist) that might hinder their performance on the upcoming test. Following existing protocols [11, 20-22], participants were given a list of commonly cited impediments used as self-handicaps in sport. To avoid exposing the nature of the dependent variable to participants, the checklist was titled the Situational Impediments Checklist (SIC). Following existing protocols [20-22], each impediment had two possible responses (Yes or No). Second, participants were asked to think about their selected impediments and estimate how much disruption their selected impediments would cause to their upcoming performances. Thus, the ratings of disruption to performance served as the measure of self-handicapping. Performance disruption was measured on a continuous scale with anchors at 1 (no disruption) and 100 (total disruption).

\subsubsection{Level of self-esteem (LSE).}

To obtain a measure of self-esteem, participants completed the Coopersmith self-esteem inventory (CSI) [23]. This inventory is reported to be a reliable and valid measure of young people's global feelings of self-worth and self-acceptance [21]. The inventory consists of 25 items relating to three areas which are answered on a yes/no scale: (1) global self-esteem: "I can make up my mind without too much trouble," and "I often wish I were someone else"; (2) relations with parents, "My parents usually consider my feelings," and "My parents expect too much of me"; and (3) relations with peers, "I'm popular with kids [of] my own age," and "Most people are better liked than I am." Self-esteem scores were calculated from the aggregate item score, with each item multiplied by 4 with higher scores reflecting higher self-esteem.

\subsubsection{Certainty of self-esteem (CSE).}

The certainty of level of self-esteem inventory contained 25 statements that corresponded with the 25 items on the CSE [23] inventory. Previous researchers [5] have used a similar procedure to obtain a measure of self-esteem certainty. For each item on the CSE, participants rated how certain they were of their responses. Participants recorded their certainty on a continuous scale with anchors at 1 (not certain at all) to 5 (very certain). Summing of all scores gave a single measure with a possible range of 25 to 125 , with higher scores indicating higher feelings of certainty.

\subsubsection{Measure of trait self-handicapping.}

The Self-handicapping scale (SHS) [24] was used to measure trait self-handicapping. The SHS contains 14 items presented on a 6-point scale with anchors at 0 (disagree very much) to 5 (agree very much). The SHS measures trait self-handicapping on two factors, trait excuse-making (TEM) and trait effort-expended (TEE) [21]. The TEM subscale reflects the proclivity to use self-handicaps that are similar to excuses. The TEM subscale is derived from nine items of the SHS and reflects an individual's proclivity to use impediments that could negatively affect performance. The TEE measure is derived from the remaining five items of the SHS and reflects the tendency to express concern about levels of effort or motivation in the preparation for performance. Higher scores on both indices represent an increased proclivity to use self-handicapping. Rhodewalt [23] reported that the two indices have acceptable validity and reliability $\left(\alpha_{T E M}=0.74, \alpha_{T E E}=0.72\right)$.

\subsection{Procedures}

At a predetermined time and date, students met with the researchers to complete the sham physical skill test. All testing sessions occurred in a private an area where no other individuals could view their performances. All participants received a package of inventories titled Feelings about Sport Questionnaire, which contained demographic self-report items, the pre-test measure of certainty the LSE, CSE, TEM, and TEM All participants completed these inventories before starting the first test. To standardize the testing protocol, a prepared script of instructions and information was read aloud to all participants. All participants were given differential information about the test.

All participants received a demonstration of how to complete the test. All participants then completed the first manipulation measure before beginning the first test. Immediately after the first test all participants received either their performance scores (non-contingent failure) or no information about their performance (non-evaluative 
condition). All scores were deceptive. Participants received their deceptive first test performance scores on a piece of paper marked "Official Test Result". The result sheet also contained the age and gender relevant percentile scores for the participants. After the first test, all participants were informed that they would have a five-minute rest period before completing the second test. After the five-minute break the researchers, instructed participants that the second trial would begin. Before starting the second trial the researchers asked participants to complete the SIC and the post measure of certainty Once the SIC had been completed the trial stopped and all participants entered the debrief phase. In this section participants were told of the deceptive manipulation that they had been exposed to and the reasons for using it. No student or parent contacted the researchers or made representation to with concerns regarding the conduct of the study.

\subsection{Experimental Design}

The design of this study involved only assessing the dependent variable (self-handicapping) after test A and the performance manipulation and then comparing the use of self-handicapping to a control group. Thus it is a post-test only control group design [25]. The rationale for such a design was premised on the needs argued by Gay [25]. First, as the study involved manipulation of individuals' perceptions of performance certainty through deception, the timeline for the study needed to be short to minimize any adverse impact. Second, assessment of initial knowledge of the dependent variable (self-handicapping) was not essential for the outcomes of the study, as the purpose of the study was to measure the effect of evaluative threat on self-handicapping. Finally, a pre-test of self-handicapping strategy might have given a false indication regarding the effect of reinforcement histories on the use of self-handicapping.

Gay [25] argued that to overcome the limitations of a post control only design, the protocol should include random sampling and a control group to control threats to validity. The use of a random age sampling process and a control group served to control for all sources of internal validity except mortality. As the length of the interaction with students was held at two weeks, with the actual manipulation and data collection occurring in one testing session, mortality was limited as a potential threat.

In this study, one level of feedback contingency and one non-evaluative condition were used based on previous research [4]. Participants were randomly assigned to either condition by using a computer random number generator. The use of sham test and bogus performance scores poses a threat to external validity and some have suggested alterations to alleviate these threats $[14,26]$. Varieties of deceptive feedback manipulations have been associated with a willingness to use mitigating excuses, which allows individuals to explain their poor performances to non-ability factors $[14,26]$. Yet, the use of heavy deception through performance scores, which suggest total failure, poses a threat to external validity. It is suggested [14] that bogus performance scores, which indicate performance outcome is equal to the $25^{\text {th }}$ percentile enables participants view feedback to be veridical and creditable. In the feedback condition (FC), the first group (non-contingent failure), participants received bogus performance scores that indicated that their ability score on the sham test was equal to the 25th percentile for their age and gender group. The second group (control-non-evaluative), participants received no information regarding their performances. Feedback contingency condition was dummy coded, ( $1=$ non-contingent failure and $0=$ non-evaluative control).

\subsection{Statistical Analysis}

Means, standard deviations and effect sizes were calculated for all LSE, CSE, TEM, TEE, and performance certainty and performance disruption. Several statistical procedures were used to meet the research aims. As the creating of performance uncertainty was critical to this study we used an analysis of covariance (ANCOVA) to assess if there was a significant difference for performance certainty between experimental and control groups before the second test, with the first measure of performance certainty used as the covariate. If the experimental manipulation was successful $(p<0.05)$, then testing of the significance of the predictor independent variables would proceed with multiple regression.

To assess the research hypothesis, a hierarchical multiple regression analysis was used to estimate the relationship between the independent factors and the dependent variable of performance disruption. Given that the relationship between the independent variables and the dependent variable was unknown, a conservative relationship was factored into the regression equation. An a priori sample size estimate of 160 indicated adequate power $(0.80)$ with five independent variables to detect $R^{2}$ values of 0.12 $(p<0.05)$ [27]. This sample size was manipulated to maintain equal cell sizes $(n=20)$ for each age and gender group.

\section{Results}

\subsection{Validation of Performance Uncertainty}

Before the first test, participants in both conditions reported similar levels of certainty (non-contingent failure condition, $M=3.46, S D \pm 0.62$; control condition, $M=3.27$, $S D \pm 0.65$ ). After exposure to the feedback contingency manipulation, there was a significant difference in certainty $\left(F(91,157)=44.29, p<0.05, \eta^{2}=0.29\right)$, with participants in the non-contingent failure group $(M=2.58, S D \pm 0.12)$ reporting less certainty before the second test than participants in the non-evaluative control group ( $M=3.30, S D \pm 0.32)$. Given that there was a significant difference between groups with the 
manipulation decreasing performance certainty for the experimental group, the regression analysis could proceed.

\subsection{Perceived Impediments to Performance}

As with previous sport based self-handicapping studies [i.e., 1,4, 11], young people cited numerous events as potential impediments to their performance. In total, 148 participants generated 420 responses, with the remaining 22 participants not citing any impediments. For the present study, the most frequently cited event or impediment was related to tiredness $(n=49)$, followed by problems with friends $(n=41)$, and an inability to concentrate $(n=40)$. Similarly, problems with playing too much sport $(n=33)$, and sports injuries $(n=23)$ were frequently cited reasons for why performance might be impaired. Events with low frequency responses included items such as, I've not felt like training $(n=3)$, I've quit sport ( $n=3)$, I've partied too hard $(n=5)$, I've had late nights $(n=6)$, and I've experienced personal problems $(n=5)$.

\subsection{Performance Disruption}

Participants across both performance manipulation conditions reported that their cited impediments would adversely affect the upcoming performances and where able to give a quantitative score to the amount of disruption the impediments would create to performance on test B. Mean scores for those who were in the non-contingent failure feedback condition were $(M=42.65, S D \pm 8.39)$ and those in the non-evaluative control condition $(M=8.85, S D \pm 6.93)$.

\subsection{Regression Estimates}

A hierarchical multiple regression analysis was used to examine the unique contribution of non-contingent failure, LSE, CSE TEM and gender. Reliability for all items were within critical threshold values except for the TEE, which had low reliability $(\alpha=0.34)$, thus it was excluded from any regression analysis. Variables proposed to explain performance disruption were entered in three steps. In step 1, performance disruption was the dependent variable and feedback manipulation was the independent variable. In step 2, CSE scores were entered into the step 1 equation. In step 3, the scales of LSE, TEM and gender were entered into the step 2 equation. All data met the assumptions for regression, with all results within specified critical threshold levels $[16,17]$.

The result for step 1, indicated that feedback contingency condition contributed to adjusted $R^{2}=0.14$, which was significantly different from zero, $F(1,158)=26.87, p<0.05$. In step 2, certainty of self-esteem variable was added to the step 1 regression equation. The change in variance accounted for was equal to 0.15 , which was not significantly different from zero $F_{\text {change }}(1,157)=3.10, \quad p>0.05$. The unstandardized regression coefficients (B),the standardized regression coefficients ( $\beta$ ), for the full model are reported in Table 1.

Table 1. Unstandardized Regression Coefficients (B) and Intercept, the Standardized Regression Coefficients ( $\beta$ ), for Variables as Predictor of Performance Disruption $(N=160)$.

\begin{tabular}{ccccc}
\hline Model & Mean & $\begin{array}{c}\text { Standard } \\
\text { deviation }\end{array}$ & $\mathrm{B}$ & $\beta$ \\
\hline $\begin{array}{c}\text { Intercept } \\
\frac{\text { Step 1 }}{\mathrm{FC}^{\wedge}}\end{array}$ & $\begin{array}{c}1(\mathrm{n}=80) \\
0(\mathrm{n}=80)\end{array}$ & & $28.40^{*}$ & \\
$\frac{\text { Step 2 }}{\text { CSE }}$ & 90.73 & \pm 8.31 & 0.62 & $0.33^{*}$ \\
$\frac{\text { Step 3 }}{\text { LSE }}$ & 48.57 & \pm 9.12 & -0.27 & $0.19^{*}$ \\
TEM & $\begin{array}{c}21.26 \\
1(\mathrm{n}=80)\end{array}$ & \pm 6.72 & -0.24 & -0.17 \\
Gender & & -0.11 & -0.03 \\
\hline
\end{tabular}

Note. Step $1 R^{2}=.14 ; \Delta R^{2}=.14(p<0.05)$

Step $2 R^{2}=.16 ; \Delta R^{2}=.15(p>0.05)$

Step $3 R^{2}=.20 ; \Delta R^{2}=.18(p<0.05)$

$\wedge$ coded as $1=$ failure and $0=$ control

$\wedge \wedge$ coded as $1=$ male and $2=$ female

$* p<0.05$ In step 3, LSE, TEM, and gender were added to the step 2 regression model. The change in variance accounted for 0.18 and was significantly different from zero $F_{\text {change }}(3,154)=2.90, p<0.05$. Level of self-esteem was the only statistically significant independent variable, $\beta=0.19$, $t(153)=-2.19, p<0.05$ A check of the confidence intervals for $B$ for TEM and gender showed that for each variable, the intervals did include zero confirming that both variables are independent of performance disruption. The variables of CSE, TEM, and gender did not significantly add to the prediction of performance disruption. As feedback contingency was dummy coded, an examination of $\beta$ indicates that non-contingent failure feedback combined with low self-esteem were significant predictors of performance disruption in a sport testing situation when low scores were meaningful.

\section{Discussion}

The purpose of this study was to explore the relationship between self-esteem and self-handicapping. The results of this investigation make it apparent that the tendency to self-handicap, particularly when failure causes uncertainty about successful performance, is closely associated with level of self-esteem. Hence, it seems reasonable to conclude that Tice's [8] contention remains tenable. Specifically, that level of self-esteem is related primarily to greater self-handicapping following failure, but not following non-evaluative circumstances. Moreover, this tendency for low self-esteem individuals to self-handicap when under evaluative threat is equal for both males and females, which is consistent with previous sport related self-handicapping research [i.e., 1, 11]

Tice's [8] contention was that self-handicaps could serve 
to enhance or protect one's self-esteem, depending upon whether they are offered following success or failure. Self-handicaps offered following success could enhance self-esteem because they imply that any subsequent success occurs despite inhibiting factors. Conversely, self-handicaps following failure might protect self-esteem because they help to sever the link between oneself and possible flawed performance.

The pattern of self-handicapping that emerged among young people in this study can be understood in light of Tice's [8] research that showed low self-esteemed young people self-handicap when esteem is threatened by a meaningful low score. The self-handicap, rather than provide opportunities for self-presentation, buffers self-view of ability from failure estimates. In Tice's [8] contention, self-handicaps protect self-esteem because they sever the link between ability and performance, therefore people with low self-esteem self-handicap to protect their self-image. The results from the present study suggest that people with low self-esteem might self-handicap when they experience failure to protect further threats to their already low self-esteem.

People with low self-esteem hold positive self-view but feel they do not have the resources to maintain highly positive images [8]. A failure experience serves to remind these people of their limited resources. In this study, young people with low self-esteem did not engage in substantial self-handicapping when they were in the non-evaluative situation. The possible explanation is that the non-evaluative environment did not contain a sufficient level of threat to warrant self-handicapping. Self-handicapping theory [2] stipulates that people only handicap in evaluative situations and that when there is an absence of diagnostic information about ability; people have no need for the strategy.

Conversely, the pattern of self-handicapping that emerged for young people with low self-esteem under conditions of failure was quite different. These young people did engage in substantially higher levels of self-handicapping when a low score was meaningful. Possibly, the evaluative situation in this study held sufficient threat because the first failure experience was a reminder to these young people that they possibly did not have personal resources to maintain a positive self-image. Subsequently, they held higher expectancies of failure on the parallel test. The threat of a second failure would confirm young peoples' fears that their abilities were insubstantial. Under such circumstances young people might have averted the negative implications of possible failure by self-handicapping.

In relation to the contribution of level of self-esteem, the present finding is not consistent with Rhodewalt's [24] contention that when people with low self-esteem have their negative views confirmed they have no need to self-handicap because they have no positive image to protect. One likely explanation for the inconsistency is that the short temporal state of failure in this study might represent a different type of evaluative threat than that of persistent failure. For example, persistent failure would confirm to people with low self-esteem that they did not have the resources to maintain their positive self-views. Nevertheless, single bouts of failure do contain threat but individuals can allay this threat with a short duration type of self-handicap such as a self-avowal.

A related explanation for the inconsistency with Rhodewalt's [24] contention was the evaluative outcomes of the test had high ego-relevance for all participants. Ego-relevance measures have not occurred in some of the previous literature. $[1,11]$ When evaluative outcomes have high ego-relevance, that is the domain has high self-relevancy, young people with low self-esteem focus not only on protecting their images, but also on protecting themselves from the attributions that can be drawn from their performance. That is, they self-handicap to protect against failure. The implication is that when young people who have low self-esteem engage in tasks that are highly self-relevant, they will adopt self-defensive behaviors if they suspect that failure is a possible outcome.

There are several implications for teachers and coaches of young people involved in sport and physical activity. Specific instances of failure are likely to be particularly threatening to young people with low self-esteem when they engage in highly self-relevant tasks. In Australia, schools use various sport talent identification tests and associative normative data during physical education. More recently, with growing community concerns about overweight school children, there is even more scrutiny and hence individual comparisons against normative data. In response, the results of this study indicate that young people who have low self-esteem are especially prone to self-handicap to attempt to sever the link between ability and possible flawed performance, when low scores have meaning. In physical education classes and sport, failure is conspicuous thus; teachers and coaches need to adopt strategies to minimize the impact of failure. The situational circumstances of attempting tasks in physical education classes ensure that performance outcomes will be largely public. Teachers and coaches need to adopt strategies that ensure that implications of failure are lessened. For example, teachers and coaches need awareness of therapeutic and counseling techniques such as minimizing the public nature of performance, the emphasis placed on success, and de-emphasizing the importance attached to failing, as a ways of lessening the evaluative threat of attempts at physical tasks.

Educators and coaches also need to be aware that self-handicaps are strategies that young people use when they suffer uncertainty about successful performance. Awareness by educators that the use of self-avowals by young people can be more than just a 'hedge betting strategy' will allow them to use proactive strategies to change the performance climate.

The inclusion of certainty of self-esteem and other self-report measures such as TEM in the regression analysis yielded some interesting findings. Self-handicapping theory and research hold that uncertainty is a key component. The contention of Harris and Snyder [5] is that certainty of self-esteem affects the tendency to self-handicap. 
Specifically, people who are uncertain of their estimations are more prone to self-handicap than people who are certain. It should be noted nonetheless that the present data do not allow a determination to be made for uncertainty of self-esteem. In this study, certainty of self-esteem did not account for a significant portion of the variance in self-reported performance disruption. There are several possible interpretations for the result.

One interpretation is that people might or might not be internally aware of how uncertain or certain their self-esteem is. This suggestion is not unique and has been noted by others [13]. For example, it is possible that the process associated with determination of certainty of self-esteem operates primarily at a non-conscious level and that some individuals are unable to accurately estimate their certainty level. Moreover, estimates of certainty of self-esteem fluctuate depending upon circumstances. Given that the measure of certainty of self-esteem occurred within a short timeframe, repeated measures might have resulted in a stronger relation to self-handicapping.

As for the remaining variables used in the regression analysis, gender did not account for the variance in self-reported performance disruption. This result is consistent with previous studies in the physical domain, which consistently show no differences in the use of self-handicapping because of gender [11]. Differences in gender might emerge for different achievement domains and variances in the self-relevancy of outcomes. In this study, the relevancy of the domain was controlled, with those who indicated that the domain has low relevance being excluded from the study. Thus, as the task was equally self-relevant to males' and females' self-concepts, it is not surprising that both self-handicapped following failure. Also, the type of self-handicapping in this study was a self-avowal, whereas gender differences are more common in the use of behavioral self-handicaps [18]. In sport, the use of self-avowels is common and thus both males and females exposed to this culture Moreover, the present results apply only to the condition of failure. Evaluative threat stemming from unexplained success might result in a different relationship between self-esteem and self-handicapping.

Also of interest is the fact that the variable of TEM did not account for a significant portion of the variance in self-reported performance disruption. This finding is not consistent with previous research [11], which indicated a significant and positive relationship. Although there are several possible explanations for the result, the most likely interpretation is that the two factors of self-handicapping [23] had low reliability for young people.

Finally, a cautionary note regarding the implications of the findings is appropriate. Although the data suggest that level of self-esteem and failure conditions are important predictors of self-handicapping, there exists an apparent conceptual-empirical disparity. In this study, the measures of level of self-esteem and certainty of self-esteem were a global assessment. Nonetheless, the construct of self-esteem is hierarchical and multifaceted. The experimental manipulation of performance certainty was for a specific aspect of self-esteem. The sham feedback involving failure posed a threat to a first order dimension of self-esteem: physical self-worth. Additionally, the threat might have also threatened feelings held for the second order dimension of sport competency. Thus, there is a conceptual-empirical disparity because the measure of self-esteem was global but the threat might have been specific to feelings associated with first and second order dimensions of self-esteem. The possibility exists that the true relation for self-esteem and self-handicapping is obfuscated by the conceptual measurement disparity.

\section{Conclusion}

Young people are willing to use self-handicaps in a sophisticated manner when faced with evaluative threat on a self-relevant task. The use of self-handicapping is a usual ploy within an acute timeframe because it might allow individuals to continue in performances when they otherwise might not. Nonetheless continual use of handicaps can result in the handicap being incorporated as part of the self. When the handicap is incorporated it might be counterproductive because the individual might use the ploy in all evaluative situations. Thus, the handicap becomes a stable internal attribute of the person and others might judge the individual differently.

\section{REFERENCES}

[1] T.A. Ryska,, Z. Yin, \& P.D. Cooley. Effects of trait and situational self-handicapping on competitive anxiety among athletes. Current Psychology: Developmental, Learning, Personality, and Social, Vol.17, 48-56, 1998.

[2] S. Berglas. Self-handicapping and self-handicappers: A cognitive/attributional model of interpersonal self-protective behavior. In R. Hogan (Ed.), Perspectives in personality: Vol. 1 (pp. 235-270). Greenwich, CT: JAI Press, 1985.

[3] E.E. Jones, \& S. Berglas. Control of attributions about the self through self-handicapping strategies: The appeal of alcohol and the role of underachievement. Personality and Social Psychology Bulletin, Vol.4, 200-206, 1978.

[4] P.D. Cooley, Self-handicapping responses of young adults. Hawaii International Education conference, Honolulu, Hawaii, 2011.

[5] R.N. Harris, \& C.R. Snyder. The role of uncertain self-esteem in self-handicapping. Journal of Personality and Social Psychology, Vol.51, 451-458, 1986.

[6] C. Kimble, E. Kimble, \& N. Croy. Development of self-handicapping tendencies. Journal of Social Psychology, Vol.138, 524-534, 1998.

[7] M.J. Strube, \& L.A. Roemmele. Self-enhancement, self-assessment, and self-evaluative task choice. Journal of 
Personality and Social Psychology, Vol.49, 981-993, 1985.

[8] D.M. Tice. Esteem protection or enhancement? Self-handicapping motives and attributions differ by trait self-esteem. Journal of Personality and Social Psychology, Vol.60, 711-725, 1991.

[9] D.M. Tice, \& R.F. Baumeister. Self-esteem, self-handicapping, and self-presentation: The strategy of inadequate practice. Journal of Personality, Vol.58, 443-464, 1990.

[10] C.R. Snyder, \& R.L. Higgins. Excuses: Their effective role in the negotiation of reality. Psychological Bulletin, Vol.104, 23-35, 1988.

[11] H. Prapavessis, \& J.R. Grove. Self-handicapping and self-esteem. Journal of Applied Sport Psychology, Vol.10, 175-184, 1998.

[12] D.B. McFarlin, \& J. Blascovich. Effects of self-esteem and performance feedback on future affective preferences and cognitive expectations. Journal of Personality and Social Psychology, Vol.40, 521-531, 1981.

[13] M.H. Kernis, D.P. Cornell, C. Sun, A. Berry, \& T. Harlow. There is more to self-esteem than whether it is high or low: The importance of stability of self-esteem. Journal of Personality and Social Psychology, Vol.65, 1190-1204. 1993.

[14] T. Thompson. Remote associate problems in performance feedback paradigms. Personality and Individual Differences, Vol.14, 11-14. 1993.

[15] S. Berglas, \& E.E. Jones. Drug choice as a self-handicapping strategy in response to noncontingent success. Journal of Personality and Social Psychology, Vol. 36, 405-417, 1978.

[16] R.L. Higgins, \& R.N. Harris. Strategic "alcohol" use: Drinking to self-handicap. Journal of Social and Clinical Psychology, Vol.6, 191-202, 1988.

[17] C.R. Snyder, T.W. Smith, R.W. Augelli, \& R.E. Ingram. On the self-serving function of social anxiety: Shyness as a self-handicapping strategy. Journal of Personality and Social Psychology, Vol.48, 970-980, 1985.

[18] S.M. McCrea, E.R. Hirt, \& B.J. Milner. She works hard for the money: Valuing effort underlies gender differences in behavioral self-handicapping. Journal of Experimental Social Psychology, Vol. 44, 292-311, 2008.

[19] D. Ulrich. Test of Gross Motor Development. Austin, TX: Pro-ed. 1985.

[20] A. Carron, H. Prapavessis, \& J.R. Grove. Group effects and self-handicapping. Journal of Sport \& Exercise Psychology, Vol.16, 246-257, 1994.

[21] H.A. Hausenblas, \& A.V. Carron. Group cohesion and self-handicapping in female and male athletes. Journal of Sport \& Exercise Psychology, Vol.18, 132-143, 1996.

[22] T.A. Ryska, Z. Yin, \& M. Boyd. The role of dispositional goal orientation and team climate on situational self-handicapping among young athletes. Journal of Sport Behavior, Vol.22, 410-425, 1999.

[23] S. Coopersmith. SEI: Self-esteem inventories. Palo Alto, CA: Consulting Psychologists Press, 1990.

[24] F. Rhodewalt. Self-handicappers: Individual differences in the preference for anticipatory, self-protective acts. In R. L. Higgins, C. R. Snyder, \& S. Berglas (Eds.), Self-handicapping: The paradox that isn't. (pp. 69-106). New York: Plenum, 1990.

[25] L.R. Gay. Educational research: Competencies for analysis and application. Englewood Cliffs, NJ: Merrill, 1996.

[26] T. Thompson. Underachieving to protect self-worth. Theory, research, and interventions. Sydney: Ashgate, 1999.

[27] J.F. Hair, R.E. Anderson, R.L. Tatham, \& W.C. Black. Multivariate data analysis (4th Ed.). Upper Saddle River, NJ: Prentice Hall, 1995. 